\title{
APLICAÇÃO DA JANELA DE JOHARI EM UMA INTERAÇÃO
}

\author{
Elizabete Regina Araújo Oliveira * \\ Maria Márcia Bachion ** \\ Emília Campos de Carvalho**
}

OLIVEIRA, E. R. A.; BACHION, M. M. ; CARVALHO, E. C. de. Aplicação da janela de johari em uma interação. Rev.latino-am.enfermagem, Ribeirão Preto, v. 4, nº especial, p. 113-25, abril 1996.

Este trabalho propõe verificar a viabilidade da Janela de Johari em encontro a dois. Metodologicamente constou das seguintes etapas: compreensão do modelo teórico da Janela de Johari; estabelecimentos de critérios para realização de uma entrevista seguindo o referencial; realização de uma entrevista com cliente que desconhece o referencial e; análise e interpretação dos resultados à luz do referencial teórico. Os resultados obtidos permitiram analisar o comportamento apresentado durante a entrevista tanto do entrevistado como do entrevistador, bem como, estabelecer parâmetros para se verificar o quanto se desconhece de uma pessoa, pelo que ela se deixa revelar, e ainda as formas que a pessoa utiliza para revelar a sua personalidade. A janela facilita visualizar a maneira como as pessoas se revelam.

UNITERMOS: interação

\footnotetext{
* Aluna do Doutorado Interunidades da Escola de Enfermagem de Ribeirão Preto da Universidade de São Paulo. Docente da UFES

** Docentes da Escola de Enfermagem de Ribeirão Preto da Universidade de São Paulo
} 


\section{INTRODUÇÃO}

O homem, desde o seu nascimento, utiliza o processo de comunicação para se fazer entendido quanto às suas necessidades. O processo se dá através da emissão de signos padronizados que são codificados pelo outro, permitindo compreensão mútua. A mente humana apresenta a capacidade de evoluir no emprego dos signos, os quais passam a ter certos conteúdos de informações, de idéias, pensamentos, intenções, desejos e conhecimentos.

Com isto, observa-se uma evolução do homem, no que tange ao processo de comunicação. O ser humano tem buscado formas de melhor se fazer entender pelo outro, de se revelar e compreender aquilo que lhe é revelado.

O presente estudo aborda uma das teorias que analisa formas de se revelar na comunicação. Trata-se de estudar a Janela de JOHARI e aplicá-la a uma situação interacional, tendo como objetivo verificar a viabilidade de aplicação da janela de johari em um encontro a dois.

\section{MODELO TEÓRICO}

A comunicação é o processo pelo qual as pessoas tentam expressar o que pensam e o que sentem para outros, como também receber reciprocamente.

Para FRITZEN (1992) a comunicação é um processo de dar e receber feedback. Para ilustrar esse processo, Joseph Luft e Harry Inghan elaboraram um modelo denominado Janela de Johari. Estes pensadores são considerados por LITTLEJOHN (1978), como pertencentes à Escola Humanista da Psicologia. Ao apresentarem a Janela de Johari como modelo de interação humana, eles procuraram desenvolver formas de uma melhor comunicação, ou seja, aperfeiçoar o processo de revelação e compreensão.

A Janela de Johari vem sendo estudada e aplicada na relação interpessoal muito mais em grupos maiores do que em grupos de duas pessoas. Para este trabalho, propôs-se aplicá-la numa relação interpessoal em nível de duas pessoas e procurou-se analisar como esse processo se efetiva de fato. Para tanto categorizou-se a comunicação, a partir dos pressupostos teóricos estabelecidos para o emprego desse referencial.

Vários autores vêm descrevendo como teoricamente é compreendido esse modelo de interação humana. Entre eles, destacam-se LITTLEJOHN (1978); FRITZEN (1992) e CARMAGNANI \& DANIEL (1992). Há unanimidade em afirmarem que esta teoria esta representada esquematicamente, por um quadrado. 
FIGURA 1 - JANELA DE JOHARI

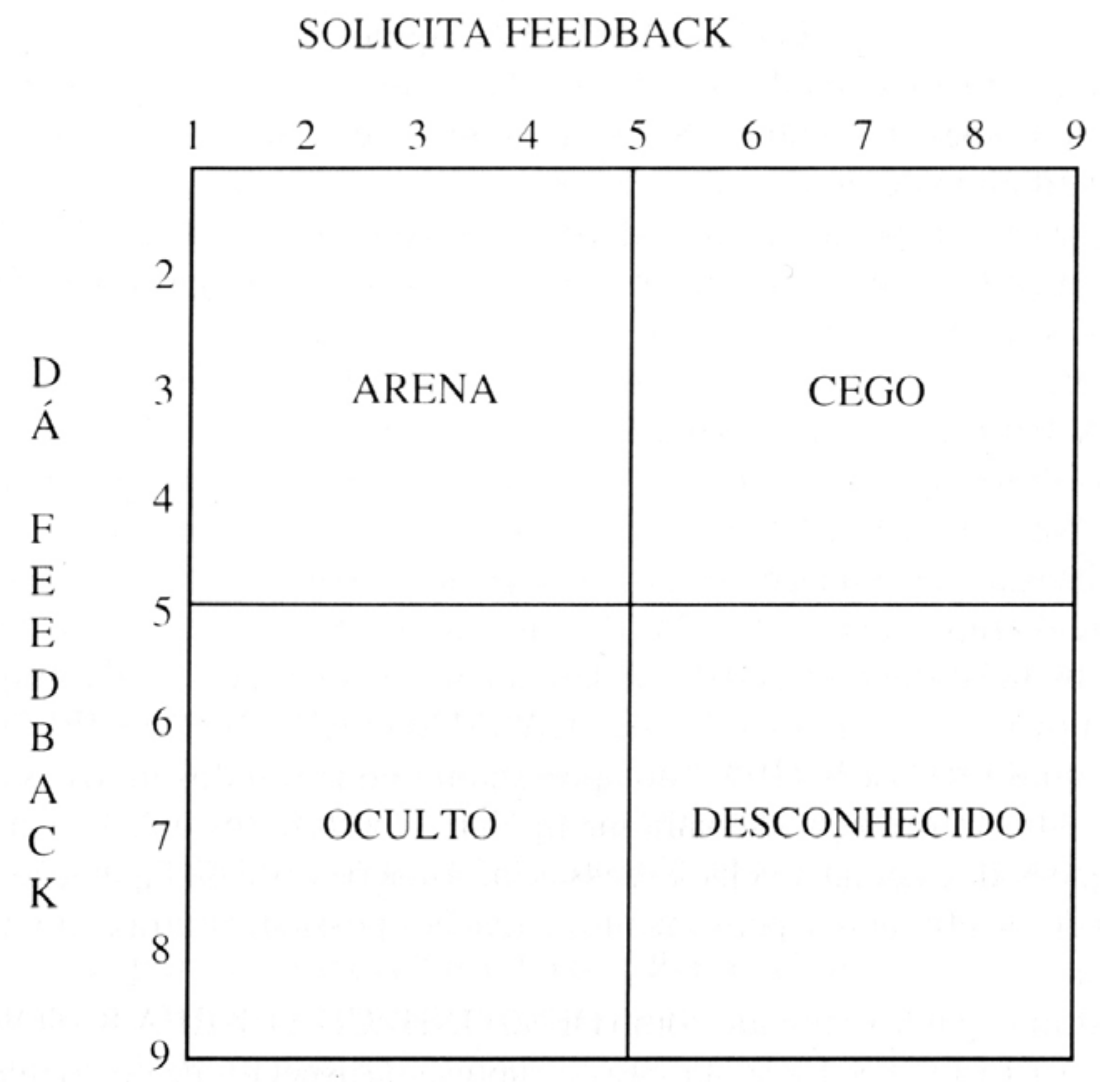

FRITZEN (1992) divide-o em áreas ou quadrantes através de colunas (verticais e barras horizontais). As duas colunas representam o eu e as duas barras representam o grupo. Essas colunas ou barras não são fixas, elas podem se deslocar e apresentar formas diferentes dos quadros desse quadrado (no seu interior), de acordo com o grau de feedback que pode ocorrer durante o processo da comunicação. Essas figuras formadas representam, para LITTLEJOHN (1978), a pessoa em relação a outra, e o modelo de percepção, no qual ela se encontra podendo estar no nível de consciência, de compreensão ou revelação.

O primeiro quadro é apresentado com o nome de ABERTO, ARENA OU ÁREA LIVRE. Nesta área se encontram as experiências e os dados conhecidos pela própria pessoa e por outras pessoas. Segundo FRITZEN (1992) "é uma área que se caracteriza pela troca livre e aberta de informações entre o eu e os outros". O comportamento nesse caso é público e disponível a todos. Essa área pode aumentar de tamanho, na medida em que o nível de confiança cresce entre os partlcipantes e no caso mais informações relevantes de caráter pessoal, são compartilhadas. 
O segundo quadro localizado na parte superior é denominado de CEGO, MANCHA CEGA OU MANCHA ESCURA. Essa área é caracterizada por apresentar informações conhecidas dos outros, mas não do eu, ou seja, aquelas informações a respeito do nosso eu "que ignoramos, mas que os outros conhecem". Segundo CARMAGNANI \& DANIEL (1992) "é aquilo que os outros sabem de nós e não nos dizem. O nosso modo de agir, de falar, o nosso estilo de relacionamento suscitam impressões nos outros. Se essas impressões não nos forem por eles reveladas (verbalmente ou com atitudes e gestos), criarão em nós a MANCHA ESCURA que gera suspeita, cautela, falta de desenvoltura". Para FRITZEN (1992) "no rol dessas informações encontram-se nossa maneira de agir, nosso jeito de falar, nosso estilo de relacionamento, etc.".

O terceiro quadro é denominado de OCULTO, FACHADA OU MÁSCARA. É representado pelo que é conhecido do eu, mas não dos outros. Esta área contém informações que sabemos a nosso respeito, mas que são desconhecidas pelo grupo. Nesta FACHADA, segundo FRITZEN (1992), mantemos oculto das pessoas as coisas que conhecemos de nós mesmos. Esse ocultismo pode ser por medo de que o grupo venha saber dos nossos sentimentos, percepções e opiniões a respeito dos seus integrantes. Isto poderá acarretar uma rejeição do grupo que poderá atingir-nos de alguma forma. CARMAGNANI \& DANIEL (1992) concordam com FRITZEN (1992) ao apresentarem que um dos motivos de não revelarmos estas informações, é para manipular e controlar os outros. Um outro agravante que pode estar na revelação dessas informações está na suposição de que a revelação dos sentimentos, pensamentos e reações possa dar outra conotação de nossa pessoa.

O último quadro é tratado como DESCONHECIDO, ÁREA IGNORADA OU HIC SUNT LEONES. É aquela área que apresenta aspectos de nossa realidade pessoal dos quais não estamos conscientes e também são desconhecidos até das pessoas com que estamos em contato. Para FRITZEN (1992) este quadrante apresenta fatores da personalidade. É o quadrante das nossas motivações inconscientes. Esta área representa o nosso DESCONHECIDO OU INEXPLORADO e poderá compreender "coisas como dinâmica interpessoal, ocorrências da primeira infância, potencialidades latentes e as que ainda estão por descobrir".

LITTLEJOHN (1978) afirma que esse modelo está baseado nos oito pressupostos do comportamento humano. O comportamento deve: ser holístico; ser entendido subjetivamente; ser emocional; não apresentar fontes claras de consciência; receber influência de aceitação, conflito e confiança; ter mais aspectos no processo do que na estrutura; ser descoberto pela experiência pessoal e ser entendido na sua complexidade.

FRITZEN (1992), analisando a comunicação como uma forma de dar e receber feedback, enfatiza a importância de desenvolver atitude de receptividade, de modo a desenvolver no outro o dar o feedback e ter prontidão para receber feedback. Para que isto se torne eficaz, ele propõe que este seja aplicável, neutro, oportuno, solicitado, objetivo, direto, especifico e comprovado. 
Além desses princípios, o mesmo autor, apresenta como ponto de destaque no receber feedback a capacidade de ouvir. Esta capacidade envolve o "processo intelectual e emocional que integra dados físicos, emocionais e intelectuais na busca de significados e de compreensão", sendo este processo o que permite atingir o objetivo da comunicação, por compreender o significado da mensagem do emissor.

\section{MÉTODO}

Pretendeu-se utilizar a Janela de Johari numa relação interpessoal, para analisar O nível de revelação e compreensão que podem ocorrer durante a mesma, num período de 22 minutos, em duas sessões. Para a primeira não se estabeleceu tema específico e nem houve estruturação prévia. O diálogo foi gravado e filmado. Em seguida foi feita a transcrição da comunicação verbal e da comunicação não verbal. Nesta fase o entrevistador não teve compreensão do modelo que seria utilizado para análise deste processo.

A partir do conhecimento e da compreensão desse referencial teórico, o entrevistador estabeleceu critérios e na segunda sessão deu continuidade ao tema que surgiu na primeira sessão, tendo sido adotado o seguinte método:

\section{I - Critérios para as Entrevistas e Passos Percorridos}

1. Estabelecimento de objetivos previamente:

- compartilhou-se uma experiência de ansiedade;

- avaliou-se a capacidade de abertura e espontaneidade do entrevistado para emissão de feedback;

- avaliou-se a capacidade do entrevistador em favorecer ou estimular a emissão de feedback do entrevistado.

2. Estabelecimento dos seguintes itens:

Participantes: duas pessoas (entrevistador e entrevistado).

Período de tempo: no mínimo 20 minutos para cada sessão ou tempo suficiente para que o entrevistado consiga expor o desconhecido.

Número de encontros: no mínimo duas sessões.

Local: ambiente reservado (para não ser interrompido).

Material: duas cadeiras e material para gravação (filmagem).

3. Estabelecimento do processo

Entrevistador selecionou um entrevistado que não tinha conhecimento prévio do modelo teórico da Janela de JOHARI e pouco relacionamento com o entrevistador; entrevistador explicou o objetivo da entrevista e da gravação ao entrevistado; entrevistador redigiu as questões que norteariam a entrevista: 
- Primeira sessão: o entrevistador estabeleceu itens de identificação (apresentação, nome, local de trabalho, atividades que realizava) e permitiu ao entrevistado colocar um problema específico (como se percebia, o que o incomodava no momento, sobre o que gostaria de falar).

- Segunda sessão: o entrevistador destacou o problema surgido na sessão anterior; utilizou questões que permitiram ao entrevistado apresentar formas de trabalhar sua ansiedade.

Indagou as formas que o entrevistado solicita feedback durante o período de ansiedade; questionou sobre as formas de como o entrevistado favorece ou dá feedback no período de ansiedade; explicou a Janela de Johari e os objetivos deste exercício; apresentou cópia da Janela de Johari, explicando como o entrevistado pode se mostrar receptivo dando e recebendo feedback; solicitou ao entrevistado a análise do próprio comportamento, durante a entrevista, e a elaboração da sua janela.

\section{II - Categorias Estabelecidas para Análise das Entrevistas}

As Categorias utilizadas para este trabalho estão representadas das seguintes formas:

$A=$ Área Livre - $\quad$ A1 - utilizada quando a comunicação do entrevistado se faz conhecida pelo entrevistador.

A2 - utilizada quando a comunicação do entrevistador se faz conhecida pelo entrevistado.

B = Mancha Cega - B1 - utilizada quando o entrevistador expressa modo de agir, seus sentimentos, etc.

B2 - utilizada quando o entrevistado expressa seu modo de agir, seus sentimentos, etc.

C = Fachada $-\quad$ C1 - utilizada quando o entrevistador desconhece o conteúdo da comunicação.

C2 - utilizada quando o entrevistado desconhece o conteúdo.

$\mathrm{D}=$ Desconhecido $=$ DNV1 - utilizada quando o não verbal do entrevistador $\mathrm{e}$ desconhecido.

DNV2 - utilizada quando o não verbal do entrevistado é desconhecido.

BNVI - utilizada para o modo como o entrevistador se comunica.

BNV2 - utilizada para o modo como o entrevistado se comunica. 
Nesta experiência, adotou-se o critério apresentado por FRITZEN (1992), onde ele apresenta que a linha vertical se movimenta em conformidade com o solicitar feedback e a linha horizontal com o dar feedback. Estas linhas permitem a verificação da janela de JOHARI. Nesta forma de traçar a Janela, não há preocupação em medir o desconhecido. O que se encontra no quadrante do desconhecido é quantificado a partir do que se mostrou conhecido, durante o processo da comunicação. A janela tende a se comportar em conformidade com o que o entrevistado se deixa conhecer e se autoconhece. Quanto mais a pessoa solicita feedback mais ela se autoconhece e quanto mais a pessoa dá feedback, mais ela deixa ser conhecida por outros.

Tendo isto como base, procurou-se somar as categorias encontradas no solicitar feedback bem como também somar as categorias encontradas no dar feedback. Estabeleceu-se uma proporcional idade entre o total de categorias encontradas, com as do receber feedback e as do dar feedback. Assim, considerou-se as categorias empregadas para $A$, aquelas que tanto se comportam para dar como para solicitar feedback para $E$, as que se comportam apenas no solicitar feedback, para $C$, as que se comportam apenas para dar feedback. Procurou-se somar as categorias do receber feedback $(A+E)$ para traçar a linha vertical, e somar as categorias do dar feedback $(A+C)$ para traçar a linha horizontal.

\section{RESULTADOS}

Após a elaboração das categorias, buscou-se no diálogo as categorias criadas, a partir da compreensão do referencial teórico da janela de JOHARI. Os resultados obtidos permitiram analisar o comportamento apresentado, ou seja, como a janela do entrevistado se apresentou no momento dessa comunicação.

Antes mesmo dessa análise, submeteu-se a julgamento o processo de categorização do diálogo, através da proposta de verificação de concordância de BATISTA \& MATOS (1984). Alcançou-se o índice de concordância para E1 de 98,5\% e o índice de concordância para E2 de 95\%. Para estes autores o índice acima de $80 \%$ pode ser considerado adequado em estudo dessa natureza.

$\mathrm{Na}$ primeira sessão obteve-se um total de 52 categorias. Dessas, 50 estão no receber feedback com 96\%, e 36 no dar feedback com 69\%. Para corresponder aos índices propostos por FRITZEN (1992), foi feito uma relação de proporcional idade entre as linhas. A linha vertical correspondeu a 8.6 e a linha horizontal 6.2, (Figura 2). Isto permitiu considerar que, na primeira-sessão, o entrevistado esteve mais aberto para receber feedback, e procurou se autoconhecer melhor, revelando algo desconhecido de si próprio para o seu conhecido. 
FIGURA 2 - JANELA DE JOHARI CORRESPONDENTE A PRIMEIRA SESSÃO. ELABORADA PELO ENTREVISTADOR

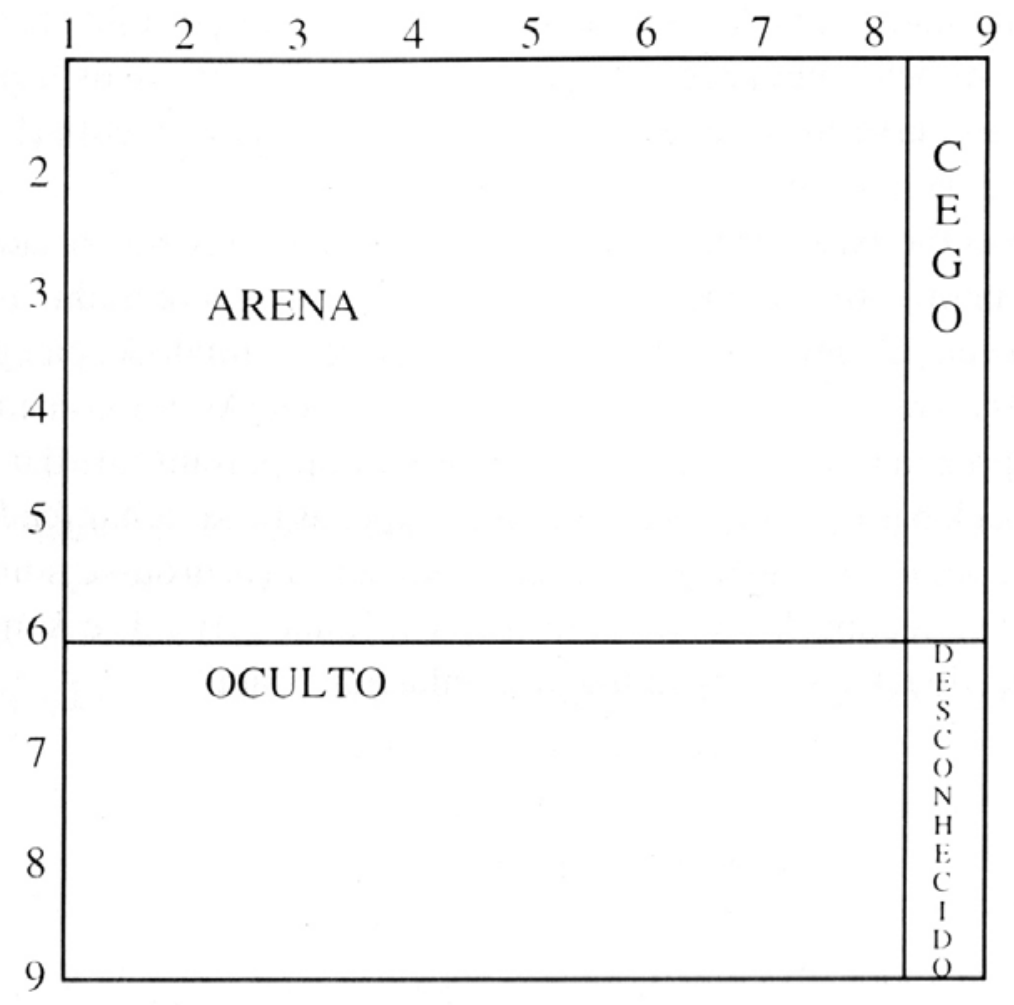

Na segunda sessão obteve-se um total de 64 categorias. Dessas, 41 estão no receber feedback com $64 \%$, e 57 no dar feedback com $89 \%$. Adotou-se o mesmo critério da primeira sessão, no que corresponde a determinação das linhas ficando a linha vertical em 5.7 e a linha horizontal em 8.0; assim foi traçada a janela para a segunda sessão (Figura 3 ). Observou-se que na segunda sessão o entrevistado esteve mais pronto a dar feedback do que receber, sendo desta forma revelado mais o desconhecido dos outros. 
FIGURA 3 - JANELA DE JOHARI CORRESPONDENTE A PRIMEIRA SESSÃO, ELABORADA PELO ENTREVISTADOR

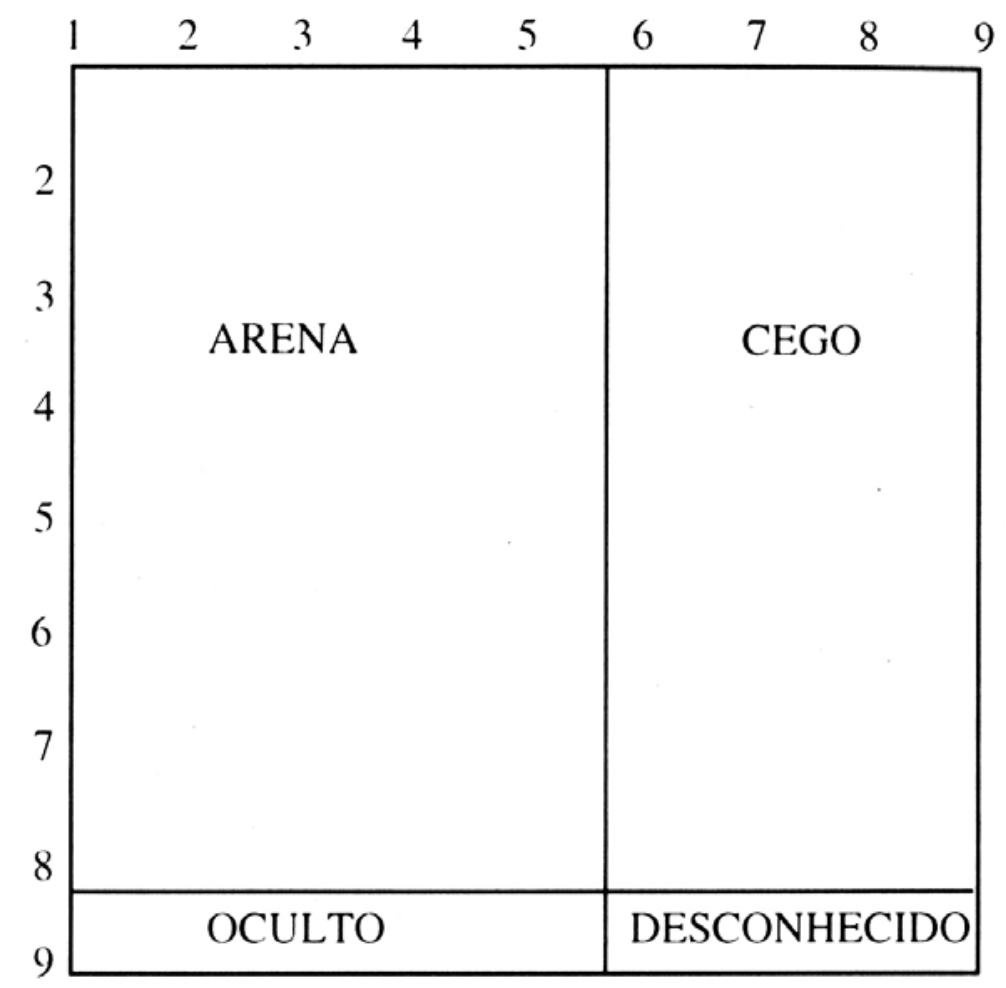

Houve uma certa dificuldade para analisar o comportamento através da Janela de Johari. Isto ocorreu por não haver acesso a alguma experiência de análise semelhante. Tomou-se conhecimento do exemplo em que a própria pessoa avalia o seu comportamento no grupo. Mediante este, ela deixa aparecer a Janela do outro no grupo, ou seja, quando ela se deixa revelar, o outro não se revela. Já neste estudo procurou-se analisar a Janela do entrevistado, o que permitiu avaliar o papel do entrevistador, ou seja, o quanto o entrevistador se mostrou terapeuta, ou o quanto ele permitiu que o outro se revelasse.

Para efeito de descrição, adotou-se o critério de FRITZEN (1992) quanto a análise das diversas posições da Janela. Em ambas, extraídas a partir das entrevistas, o comportamento do entrevistado se enquadrou no modelo que corresponde à "janela ideal" numa situação grupal ou em qualquer tipo de relacionamento significativo. Nesta, o tamanho da "Arena" aumenta à medida que cresce o nível de confiança no grupo. Os critérios desenvolvidos no sentido de dar e receber feedback facilitam este tipo de intercâmbio. A "Arena amplamente aberta indica que grande parte do comportamento de uma pessoa está liberada e aberta aos outros membros do grupo. Conseqüentemente, será menor a tendência; por parte dos outros, no sentido de interpretar (ou mal-interpretar) ou projetar significados pessoais no comportamento da pessoa" (FRITZEN, 1992). 
Em se tratando de estilos interpessoais, o mesmo autor caracteriza o uso dessa exposição e do feedback, como estilo tipo D. Este estilo enquadra um relacionamento interpessoal de abertura de feedback muito espontâneo. As pessoas usam de franqueza, permitindo abertura, o que leva a uma maior sensibilidade pelas necessidades dos outros, promovendo maior participação e maior produtividade.

Ao final da segunda sessão pediu-se à entrevistada que elaborasse a sua janela, tomando para análise o seu comportamento apresentado durante a entrevista.

A janela, conforme figura a seguir, apresenta a área de Fachada e a de Desconhecido ausentes, uma vez que a entrevistada inseriu a linha horizontal no ponto 9.0 e a vertical em 8.0. FRITZEN (1992) também não apresenta característica dessa Janela, porém a que mais se assemelha é a de estilo tipo $D$, que coincide com a janela feita pelo entrevistador, a partir da colocação do entrevistado.

\section{FIGURA 4 - JANELA DE JOHARI CORRESPONDENTE AS DUAS SESSÕES, ELABORADAS PELO ENTREVISTADOR}

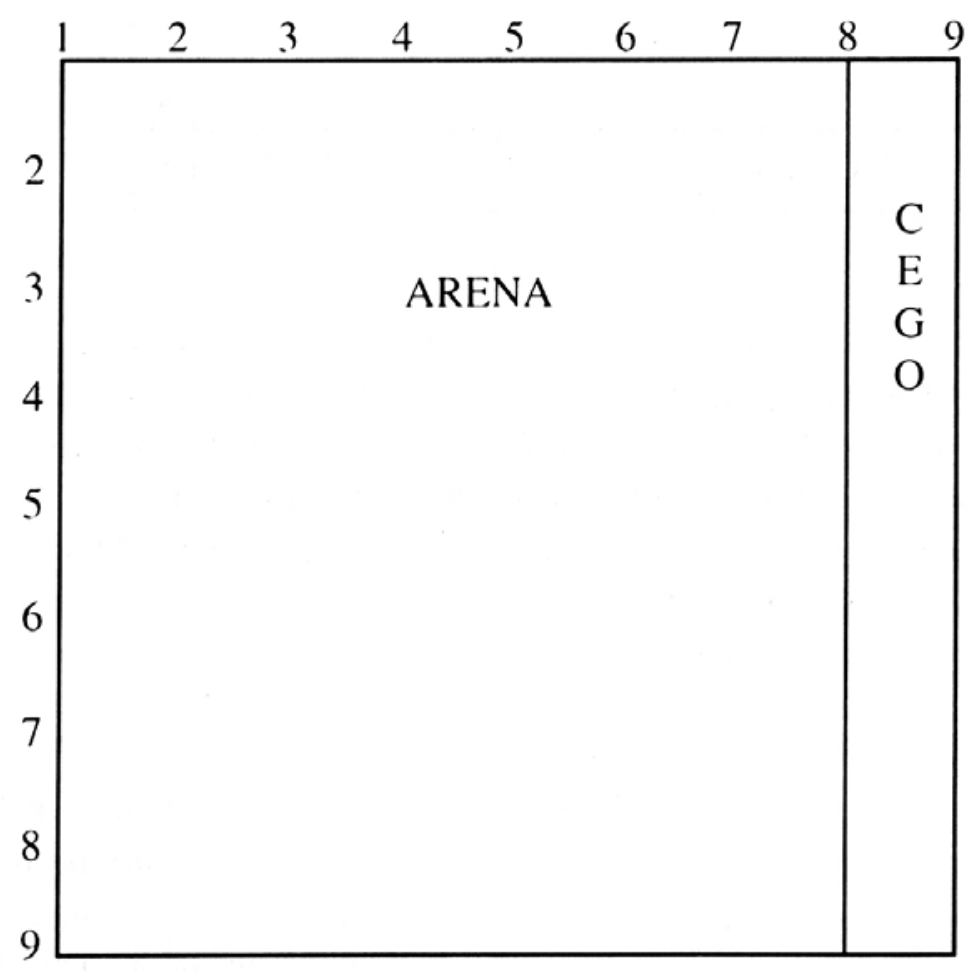

A partir da montagem dessas duas janelas concluiu-se a percepção do entrevistado em relação a sua personalidade, ou seja, o seu comportamento durante as entrevistas; ambas as janelas apresentaram-se bastante semelhantes. A área de arena esteve bem aberta, revelando-se aos outros de maneira bem espontânea e equilibrada. Diferenciou-se da elaborada pelo entrevistador nos seguintes aspectos: 
- a área da fachada não se apresentou pela abertura da área de arena, significando que ela se mostrou conhecida pelos outros mais do que por si mesma;

- a área de mancha cega sobrepôs a do desconhecido.

\section{CONSIDERAÇÕES FINAIS}

Por considerar a comunicação como um processo de dar e receber feedback, sugere-se que para se obter um melhor resultado na aplicação da Janela de Johari, ela seja aplicada em mais sessões e em período maior de tempo. Isto para que se dê tempo ao entrevistado e entrevistador de sentirem a necessidade de buscar revelar o desconhecido, mesmo porque o processo de interação tende a progredir, caso ambos consigam adquirir mais confiança e sintam a necessidade de se revelarem para compreenderem e serem compreendidos pelos outros. Tal processo permite 0 feedback proposto pelo processo da comunicação. Em uma única sessão pode-se tirar conclusões precipitadas com relação à personalidade desse indivíduo, por não ter havido oportunidade dele se revelar.

A experiência mostrou que a compreensão do desconhecido se faz de forma mais eficiente, a partir do momento em que se consegue analisar as formas ou maneiras que a pessoa utiliza para se revelar; já em uma única sessão o processo não é tão eficaz.

A janela é aplicada com o conhecimento prévio da pessoa, onde ela mesma considera sua capacidade de dar e receber feedback.

Há que se analisar mais a forma de se estruturar a janela, a partir da percepção de outros pesquisadores que testem a eficácia e a fidedignidade da .análise, a partir dessa experiência.

Sugerimos ainda, como critério para aplicação de nova experiência, a permissão de confronto; nesta, o entrevistador deve apresentar ao entrevistado a Janela de Johari, elaborada a partir dos dados do comportamento do entrevistado durante os encontros. Posteriormente, o entrevistador e entrevistado confrontam as duas janelas: a elaborada durante este processo pelo entrevistador com a elaborada pelo entrevistado. A seguir, ambos analisam e concluem qual a mais significativa, procurando destacar os itens de confronto.

Acredita-se que o trabalho só será concluído com esta etapa, pois nesta é que se dará oportunidade ao entrevistado de refletir sobre seu comportamento, a partir da compreensão da sua personalidade.

Destaca-se, por fim, um aspecto importante deste estudo, ou seja.o fato de que o enfermeiro necessita desenvolver sua capacidade terapêutica, isto é permitir ao entrevistado a verbalização dos seus sentimentos. A análise utilizando o 
referencial da Janela de Johari permite ao enfermeiro o estudo do comportamento do paciente e, de forma complementar a análise da sua capacidade terapêutica.

\section{APPLICATION OF THE JOHARI'S WINDOW IN AN INTERACTION}

The present study proposes to verify the viability of Johari's window in a twopeople meeting. Methodologically the study comprised the following stages: comprehension of the theoretical model of Johari's window establishment of criteria for the accomplishment of an interview following the reference; accomplishment of an interview with a client that do not know the reference; analysis and interpretation of the results through Johari's window. The results allowed the authors to analyze the interviewer and interviewee behavior during the interview. They also verified the amount that it is unknown about the other, through what he/she allows to he revealed and analyzed the way that a person uses to reveal his/her own personality. The window makes it easier to visualize the manner that people reveal themselves.

UNITERMS: interaction

\section{APLICACIÓN DE LA VENTANA DE JOHARI EN UNA INTERACCIÓN}

El presente estudio ha verificado la viabilidad de la ventana de Johari, en un encuentro de dos. Metodológicamente, abarcaran se las siguientes etapas: comprensión del modelo teórico de la ventana de Johari, establecimientos de criterios para la realización de una entrevista, siguiendo el referencial; realización de una entrevista con un cliente que desconoce el referencial; análisis e interpretación de los resultados, a través de la ventana de Johari. Los resultados permitieron analizar el comportamiento presentado en la entrevista, tanto del entrevistador como del entrevistado, también establecer parámetros para verificar en cuanto se desconoce una persona por parte del otro, por lo que ella se deja conocer y aún analizar las formas que las personas utilizan para revelar su personalidad. La ventana facilita la visualización de la manera como las personas revelan su personalidad.

UNITERMOS: interacción

Rev. Latino-am.enfermagem - Ribeirão Preto - v. 4 - n. especial - p. 113-125 - abril 1996124 


\section{REFERÊNCIAS BIBLIOGRÁFICAS}

01. BATISTA, C.G.; MATOS, M.A. O acordo entre observadores em situação de registro cursivo: definições e medidas. Psicologia, v. 10, n. 3, p. 57-9, 1984.

02. CARMAGNANI, R.; DANIEL, M. Ser para o outro. São Paulo: Edições Loyola. 1992. p. 32-3.

03. FRITZEN, S.J. Janela de Johari. Rio de Janeiro: Vozes, 1992. p.115. 04. LITTLEJOHN, S.W. Fundamentos teóricos da comunicação humana. Rio de Janeiro: Zahar, 1978. p. 221 -23. 